Corresponding values above the WHO threshold of $20 \mu \mathrm{g} / \mathrm{m}^{3}$ would be 51.73 attributable deaths (12.58 cardiovascular and 4.17 respiratory) 13.60 cardiac, 5.37 cerebrovascular and 49.13 respiratory hospital admissions.

Conclusions The expected exposure appears to have a limited impact on health. Future monitoring of the actual exposure levels during the progress of the works will allow evaluating the accuracy of those estimates.

\section{INTERACTION BETWEEN GENETIC AND OCCUPATIONAL FACTORS IN LUNG CANCER AETIOLOGY. A POPULATION-BASED CASE-CONTROL STUDY}

${ }^{1,2}$ Sara De Matteis, ${ }^{3}$ Melissa Rotunno, ${ }^{3} \mathrm{Kai} \mathrm{Yu}_{\mathrm{u}}{ }^{2}$ Dario Consonni, ${ }^{4}$ Roel Vermeulen, ${ }^{4}$ Hans Kromhout, ${ }^{3}$ Neil Caporaso, ${ }^{3}$ Maria Teresa Landi, ${ }^{2}$ Pier Alberto Bertazzi. ${ }^{1} /$ mperial College, National Heart and Lung Institute, London, UK; ${ }^{2}$ University of Milan and Fondazione IRCCS Ca' Granda Ospedale Maggiore Policlinico, Milan, Italy; ${ }^{3}$ National Cancer Institute, NIH, Bethesda, MD, USA; ${ }^{4}$ Institute for Risk Assessment Sciences, Utrecht University, Utrecht, The Netherlands

\subsection{6/oemed-2014-102362.105}

Objectives Genetic susceptibility in work-related lung cancer aetiology could have an important public health impact. Few studies have previously evaluated this issue, with inconsistent results. We aimed to investigate interactions between exposure to occupational carcinogens and genetic polymorphisms in lung cancer aetiology, adopting a systematic integrated approach.

Method EAGLE, a population-based case-control study, enrolled 2100 lung cancer cases and 2120 controls (Italy, 2002-2005). Lifetime work histories were collected for 4059 subjects and translated into exposure to six occupational carcinogens (asbestos, silica, polycyclic aromatic hydrocarbons, diesel exhausts, chromium, and nickel) using a job-exposure matrix. We selected 23 candidate genes among phase II metabolic genes reported in association with lung cancer susceptibility and/or metabolism of selected carcinogens. 298 tagging single nucleotide polymorphisms (SNPs) were genotyped on 4050 subjects. We tested for interaction within smoking-adjusted logistic regressions where SNPs were modelled individually, by gene group (using gene scores and haplotypes), and by pathways. False discovery rate (FDR) was used to account for multiple testing. Gene expression changes in lung tissues were studied for SNPs-carcinogens significant interactions.

Results As asbestos had the highest impact on lung cancer burden, we restricted interaction tests to this carcinogen. GSTM4 polymorphisms consistently showed positive interactions across different analysis levels, especially by SNP group score (FDRadjusted p-value for interaction $<0.0001)$. No significant genetic "signal" by asbestos exposure was found at lung tissue level.

Conclusions GSTM4 polymorphisms may play a role in asbestos-related lung cancer aetiology. These findings are biologically plausible and have never previously been reported; they should therefore be validated in further studies.

\section{ESTIMATING THE BURDEN OF OCCUPATIONAL CANCER TAKING INTO ACCOUNT AGE}

${ }^{1}$ Sally Hutchings, 'Lesley Rushton, ${ }^{2}$ Tim Driscoll. 'Imperial College London, London, UK; ${ }^{2}$ University of Sidney, Sydney, Australia

10.1136/oemed-2014-102362.106
Objectives Estimates of burden of disease are generally based on population attributable fractions (PAFs) calculated for a whole population. However, the age structure of an exposed group has an impact on these estimates, because disease rates vary by age and the exposed population may be younger than the national population in the estimation year.

Method To account for this, PAFs can be calculated by age, and applied separately by age to national incidence data. We have adapted our risk period methodology, which takes account of latency to estimate numbers exposed to a causative agent using Levin's formula for PAF, to estimate a workforce turnover factor by age group, which accounts for the age structure of an exposed population. To estimate age-specific RRs from unit relative risks per year of exposure, the link between age and duration of exposure can be modelled using MonteCarlo methods.

Results We show the effect of estimating the burden of lung cancer due to occupational exposure to respirable crystalline silica for Britain using PAF estimates which do or do not take age into account. Taking account of age and assuming recruitment between ages 15-44, there were 1188 lung cancer registrations in males in 2010, or 798 without accounting for age, or 636 vs. 804 assuming recruitment between ages $15-24$. The extension to using age-specific RRs is demonstrated for occupational asbestos-related lung cancers.

Conclusions Given the above results, and although highly dependent on assumptions made about workforce ages, there is clearly a case to be made to estimate PAFs by age.

\section{MORTALITY IN THE FRENCH COHORT OF NUCLEAR WORKERS MONITORED FOR EXTERNAL RADIATION EXPOSURE}

${ }^{1}$ Klervi Leuraud, ${ }^{1}$ Olivier Laurent, ${ }^{1}$ Eric Samson, ${ }^{1}$ Sylvaine Caër-Lorho, ${ }^{2}$ Alain Acker, ${ }^{2}$ Pierre Laroche, ${ }^{3}$ Bernard Le Guen, ${ }^{3}$ Dominique Hubert, ${ }^{4}$ David Richardson, ${ }^{1}$ Dominique Laurier. ${ }^{1} / R S N$, Fontenay Aux Roses, France; ${ }^{2}$ AREVA, Paris, France; ${ }^{3} E D F$, Saint-Denis, France, ${ }^{4}$ University of North Carolina at Chapel Hill, Chapel Hill, USA

\subsection{6/oemed-2014-102362.107}

Objectives Understanding the effect of chronic low dose radiation exposure is crucial for radiation protection. This study analyses mortality of workers monitored for external radiation exposure while employed at three major French nuclear companies.

Method The cohort includes all workers employed at least one year by CEA, AREVA NC or EDF between 1950 and 1994, monitored for radiation exposure and alive on 1 January 1968. The mortality follow-up was to 2004. Vital status and causes of death were obtained from national registries. Standardised mortality ratios were assessed using national rates as the reference.

Results A total of 59004 workers were followed-up for an average of 25 years. Mean age at end of follow-up was 56 years. Less than $1 \%$ of workers were lost to follow-up. 6310 deaths occurred between 1968 and 2004 including 2547 cancer deaths. A strong healthy worker effect was observed (all-cause SMR = 0.61, 95\%-CI: 0.60-0.63). Significant excess mortality was observed for pleura cancer $(\mathrm{SMR}=1.71,95 \%-\mathrm{CI}$ : 1.24-2.30) and for melanoma (SMR $=1.43,95 \%-\mathrm{CI}$ : 1.04-1.92), with no significant trend in SMRs for these outcomes across categories of cumulative radiation exposure.

Conclusions This analysis of French nuclear workers confirms a healthy worker effect but also an excess risk of death 